\title{
Investigation via morphological analysis of aluminium foams produced by replication casting
}

\author{
A. Boschetto, L. Bottini, F. Campana, L. Consorti \\ Dip.di Ingegneria Meccanica e Aerospaziale, Sapienza Università di Roma, Roma (Italy) \\ alberto.boschetto@uniroma1.it,luana.bottini@uniroma1.it,francesca.campana@uniroma1.it,lconsorti@gmail.com \\ D. Pilone \\ Dip. Ingegneria Chimica Materiali Ambiente, Sapienza Università di Roma, Roma (Italy) \\ daniela.pilone@uniroma1.it
}

\begin{abstract}
Foams and porous materials with cellular structure have many interesting combinations of physical and mechanical properties coupled with low specific weight. By means of replication casting it is possible to manufacture foams from molten metal without direct foaming. A soluble salt is used as space holder, which is removed by leaching in water. This can be done successfully if the content of space holding fillers is so high that all the granules are interconnected. One of the main advantages of using the replication casting is a close control of pore sizes which is given by the distribution of particle sizes of the filler material. This contrasts with the pore size distribution of the materials foamed by other processes where a wider statistical distribution of pores is found. On the other hand, the maximum porosities that can be achieved using space holders are limited to values below $60 \%$, whereas the other methods allow for porosities up to $98 \%$. Temperature of the mould and infiltration pressure are critical process parameters: a typical problem encountered is the premature solidification of the melt, especially due to the high heat capacity of the salt.

In this work foam properties such as cell shape, distribution and anisotropy and defect presence are investigated by using digital image processing technique. For this purpose replicated AlSi7Mg0.3 alloy foams are produced by infiltrating preforms of $\mathrm{NaCl}$ particles, varying the metal infiltration pressure and the mould preheating temperature.

An original procedure based on image analysis has been set up to determine size, morphology and distribution of cells. The paper demonstrates that this methodology, coupled with microstructural analysis, is a useful tool for investigating the effects of process parameters on foam properties.
\end{abstract}

KEYWORDS. Aluminium foams; Foam morphology; Image analysis; Replication casting; Watershed method.

\section{INTRODUCTION}

$\mathrm{M}$ etal foams are a class of materials with interesting physical, mechanical, thermal, electrical, structural and acoustic properties. The current understanding of production methods ranges from ancient to novel processing techniques, many of which still under development. The processes can be divided into several classes: melt gas injection, gas-releasing particle decomposition in the melt or in semi-solids, casting using a polymer or wax precursor as template, metal decomposition on cellular preforms, entrapped gas expansion, metal infiltration through a leachable pattern[1]. 
This last technique is characterised by the following steps: a bed of particles of a leachable material is infiltrated by liquid metal under pressure and allowed to cool [2], afterwards leaching of the particles gives a cellular metallic structure of great uniformity. Although salt grains offer the advantage that they can be sintered to enhance the connectivity of the salt and change the structure of the pattern, the obtainable density depends on the packing efficiency of the granules, which is related to their size distribution. This process was pioneered by Polonsky et al. [3] that produced aluminium alloy foams. Only in the recent 15 years the production of high-purity foams by replication has been revived [4]. Adair et al. [5] studied the crystal shape and the growth of salt crystals to obtain several structures. In Gaillard et al. [6] the authors set up a procedure to fabricate $\mathrm{NaCl}$ powders by controlling their shape. Foams with spherical cells have been produced by Jiang et al. [7]. Goodall et al. [8, 9] varied the foam relative density by densifying the $\mathrm{NaCl}$ preform before infiltration. The results showed that cold pressing rather than sintering yields superior Young modulus.

Despois et al. [10] investigated the effect of infiltration pressure on mechanical properties and permeability of foams. They found that as the pressure is increased, small finger-like protrusions appear lowering the foam permeability to fluid flow. On the other hand mechanical properties such as Young's modulus and yield stress increase with increasing relative density. Kadar et al. [11] studied the compression behaviour of foams manufactured by replication casting of an eutectic Al-Si alloy. The acoustic emission has been employed to study that behaviour.

Quadrini et al. [12] observed different microstructures along the height of the samples: the grain size decreases from the top, near the cooled piston, to the bottom of the foam. This behaviour can be related to the cooling rate and pressure during solidification [13]. It is noteworthy that the grain size distribution is everywhere fine if compared with the bulk sample: this is due not only to the holding pressure but also to the salt precursor. Thus good mechanical properties are expected and a further increase could be obtained after heat treatment. Porosity of the foam has been measured by Boschetto et al. [14] by means of digital image processing. The morphological analysis of the foam showed sharpened voids, especially in comparison with the ones obtained by compact powder processes. This is justified by the salt pattern that determines the formation of angular and faceted cells.

At present metal foams have a very wide range of applications due to their properties [15]. Examples are: lightweight structures due to excellent stiffness-to-weight ratio [16]; heat exchangers and refrigerators due to their high specific surface area [17]; energy absorbers for the ability to absorb energy at constant pressure [18, 19]; acoustic absorbers for their sound absorbing capacity [19]; sandwich cores due to low density and fracture strength; flame arrester panels for their conductivity; filters for gas and liquid filtration; catalyst carriers for their good conductivity and high specific surface area; shock wave dissipation devices due to dumping capacity; air batteries.

Considering that each application requires specific foam properties, knowing the relationship between manufacturing process parameters and foam properties is of paramount importance to tailor properties for a given application. For that reason in this work an original procedure based on image analysis has been set up to determine size, morphology and distribution of cells. This methodology, coupled with microstructural analysis, is a useful tool for investigating the effects of process parameters on foam properties.

In order to demonstrate the capabilities of the proposed method, in this paper the results related to twelve AlSi7 Mg0.3 alloy specimens produced by using different process parameters are discussed. First the experimental set up to produce the foams is described together with the proposed specimen analysis method, then results and discussion are reported and finally the conclusions are drawn.

\section{EXPERIMENTAL}

\section{Specimen fabrication}

he alloy used for producing foams is AlSi7Mg0.3 (A356.0). This alloy is commonly used in pressure and gravity casting for fabricating electric motors, automotive and structural components. The pattern was made of commercial $\mathrm{NaCl}$ that allows to set up a quite cheap industrial process. The mould was composed by a cylindrical core (Fig.1), a positioning base and an upper conveyor. The resulting specimen is $28 \mathrm{~mm}$ in diameter and $50 \mathrm{~mm}$ in height. A hollow oven allowed to keep constant the temperature of the model by a thermostatic controller. A hydraulic cylinder was employed to provide the pressure for the infiltration and solidification stage. The assembled apparatus is reported in Fig. 2. Pressure, piston speed, mould and crucible temperatures were acquired by an A/D input device system and controlled through Labview.

The specimen manufacturing process includes several steps. First a sample (40 g) was taken from an aluminium ingot. The alloy was put in a graphite crucible heated in a muffle at $700{ }^{\circ} \mathrm{C}$. The salt $(9 \mathrm{~g})$ was sieved by means of a $3 \mathrm{~mm}$ mesh sieve in order to eliminate all the smaller particles, placed in a second crucible and heated at $700{ }^{\circ} \mathrm{C}$. The hollow oven was used 
to keep constant the mould temperature. Then the salt was poured into the mould and subsequently the aluminium alloy was cast. The piston was pushed at a speed of $32 \mathrm{~mm} / \mathrm{s}$ to close the mould and provide the predefined pressure to infiltrate the pattern. The pressure was maintained for $30 \mathrm{~s}$. After solidification and cooling the specimen was extracted by opening the mould. Then the salt was dissolved in water. By means of this process 12 specimens were produced changing mould temperature and injection pressure on two levels: $(500,550){ }^{\circ} \mathrm{C}$ and $(20,30)$ bar.

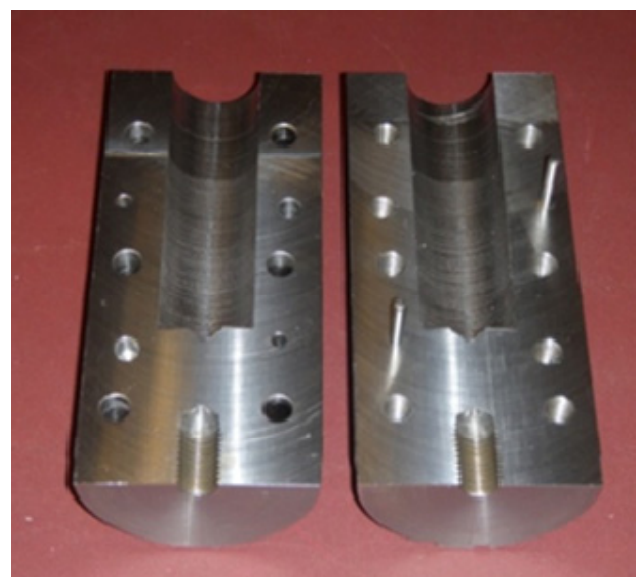

Figure 1: Mould used for foam production.
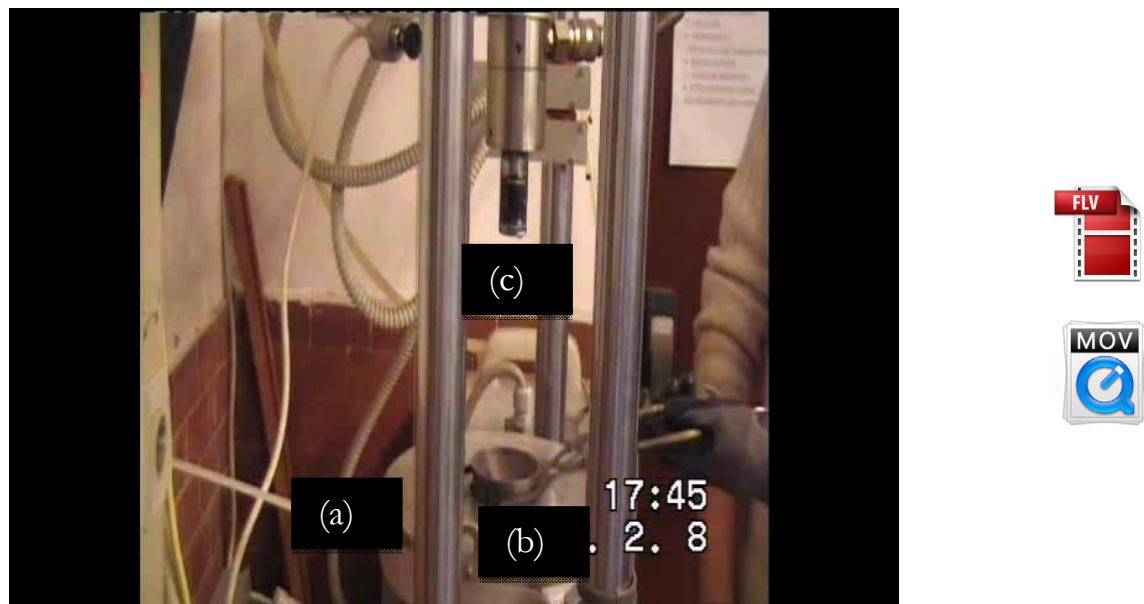

Figure 2: Experimental apparatus: (a) hollow oven, (b) mould, (c) piston.

\section{Specimen analysis}

The analysis was performed on longitudinal sections of every specimen after cutting by a Minitom Struers cut-off machine (125 $\mathrm{mm}$ in diameter and 0,5 $\mathrm{mm}$ thick wheel, with bakelite bonded $\mathrm{SiC}$ abrasive). Images of these sections were acquired in order to perform image analysis of voids. To this end the surfaces were painted using a black dye in order to obtain good contrast. The specimens were ground with a series of SiC papers and afterwards polished with $1 \mu \mathrm{m}$ alumina.

Then they were acquired by an Image Sensor Type CCD with 5.04 Megapixel resolution and $\mathrm{f} \backslash 2.8$ Carl Zeiss optic lens. The image of each specimen was calibrated to avoid aberrations and positioning errors [19]. It was enhanced by means of contrast equalisation and bright correction. A median filter was applied in order to avoid random error and impulse noise. A binarisation separated features (cell cavities) from background.

The segmentation was performed by using an automatic watershed method [14] developed to highlight each cavity and each cell contributing to cavity formation. In fact some process problems can produce larger cavities by cell intersection. The automatic watershed method allows to isolate these cells by using seeds positioned at the intersections of the segments that define the binary image skeleton (Fig. 3c and Fig. 3d). Fig. 3e shows the results of the seed definition overlapped to the original specimen image. From these seeds a segmentation of the cells that contribute to each cavity is determined (Fig. 3f). 


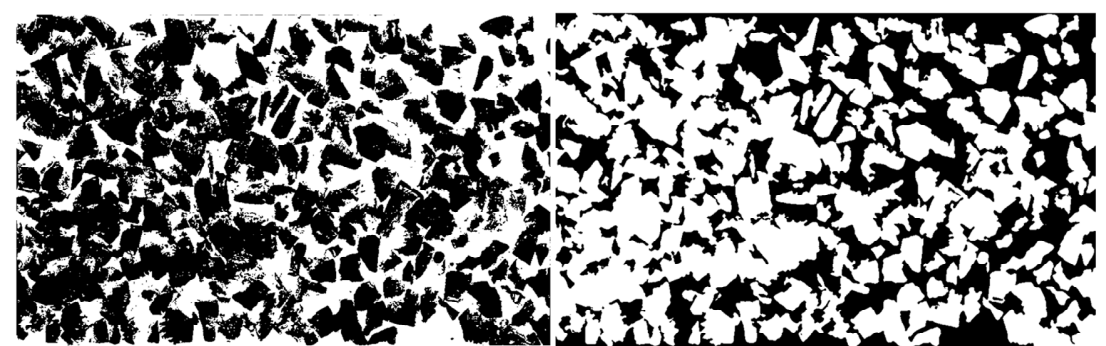

(a)

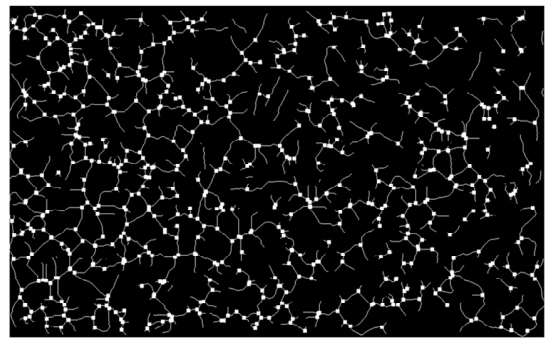

(d) (b)

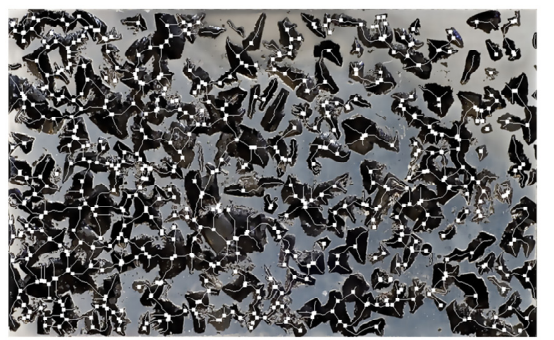

(e)

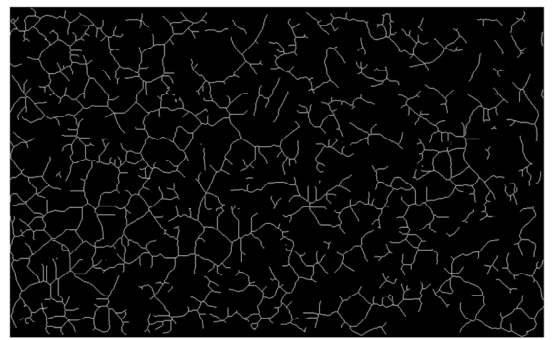

(c)

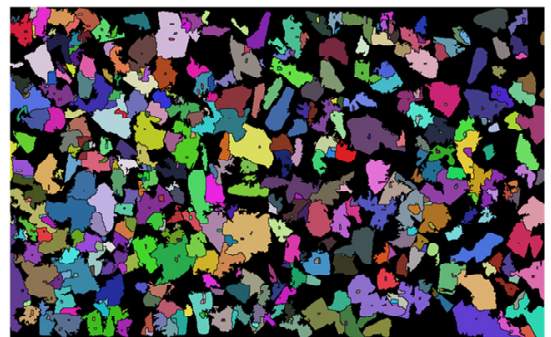

(f)

Figure 3: Automatic watershed method isolates cell contributing to cavity formation. (a-f) is the sequence of image processing from image binarisation (a) to segmentation (f).

Shape indicators considered in this study are circularity, rectangularity and elongation. Circularity is a ratio of the perimeter of a circle with the same area as the particle divided by the perimeter of the actual particle image. Rectangularity is measured by the ratio of the area of the region to the area of its minimum bounding box. Elongation is calculated as 1width/height where width is the smallest axis of the best-fit ellipse of data and height is the largest one.

\section{RESULTS AND DisCUSSION}

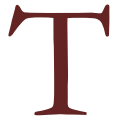
ab. 1 shows the global characteristics of the specimens in terms of weight, density and height according to process parameters. It can be observed that the mean density value is $1.045 \pm 0.110 \mathrm{~g} / \mathrm{cm}^{3}$. A close observation of the specimens reveals that four of them are characterised by a discontinuous external wall. This is related to the mould preheating temperature that affects cooling rates and that can determine a premature solidification of the foam external layer. This assumption is confirmed by the fact that four of the six specimens obtained by preheating the mould at $500^{\circ} \mathrm{C}$ have this defect (Fig. 4).

\begin{tabular}{|c|c|c|c|c|c|}
\hline Specimen & $T\left[{ }^{\circ} \mathrm{C}\right]$ & $P$ [bar] & $\begin{array}{c}\text { weight } \\
{[g]}\end{array}$ & $\begin{array}{c}\text { height } \\
\text { [mm] }\end{array}$ & $\begin{array}{c}\text { density } \\
{\left[\mathrm{g} / \mathrm{cm}^{3}\right]}\end{array}$ \\
\hline \#1 & 500 & 20 & 30.2 & 52.84 & 0.928 \\
\hline \#2 & 500 & 20 & 32.9 & 49.21 & 1.086 \\
\hline \#3 & 500 & 20 & 32.4 & 56.18 & 0.937 \\
\hline \#4 & 500 & 30 & 28.8 & 49.97 & 0.936 \\
\hline$\# 5$ & 500 & 30 & 30.7 & 45.26 & 1.102 \\
\hline \#6 & 500 & 30 & 24.9 & 44.44 & 0.910 \\
\hline \#7 & 550 & 20 & 33.5 & 48.80 & 1.115 \\
\hline \#8 & 550 & 20 & 31.8 & 52.00 & 0.993 \\
\hline \#9 & 550 & 20 & 34.1 & 47.24 & 1.172 \\
\hline$\# 10$ & 550 & 30 & 34.7 & 47.45 & 1.188 \\
\hline \#11 & 550 & 30 & 33.2 & 44.92 & 1.200 \\
\hline \#12 & 550 & 30 & 27.1 & 45.25 & 0.973 \\
\hline
\end{tabular}

Table 1: Global characteristics of the specimens. 


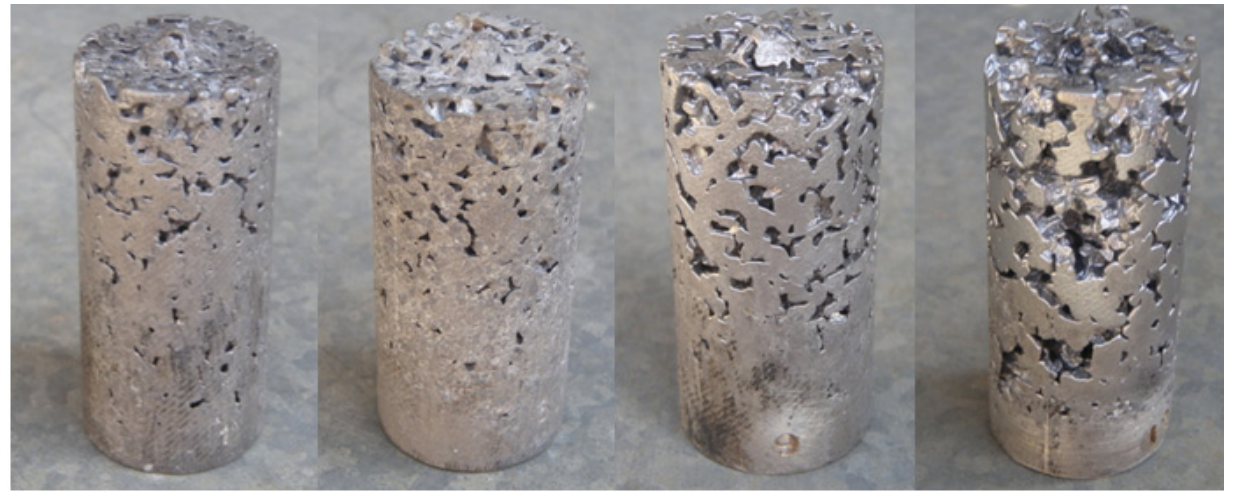

\#1

\#3

\#9

\#12

Figure 4: Macrographs showing the external appearance of specimens characterised by a discontinuous external layer.

Fig. 5 shows foam density as a function of temperature and pressure. Foam density decreases by decreasing mould temperature. In fact a mould temperature decrease raises cooling rates with consequent quick solidification of the alloy occurring probably before the mould is completely filled. This phenomenon explains also the reason why specimens \#1, \#3, \#9, \#12 have a discontinuous external layer. As far as the pressure is concerned Fig. 5 shows that it does not affect specimen density at the lower considered mould temperature while at higher temperature its increase seems to minimally raise density. This can be explained considering that at $550^{\circ} \mathrm{C}$ solidification is slightly delayed and then an higher applied pressure may enhance metal infiltration through the salt pattern. The Analysis of Variance (ANOVA) with a 95\% confidence interval, confirmed that there are no significant evidences for pressure single effect as well as pressuretemperature interaction.

\section{$500^{\circ} \mathrm{C} \square 550^{\circ} \mathrm{C}$}

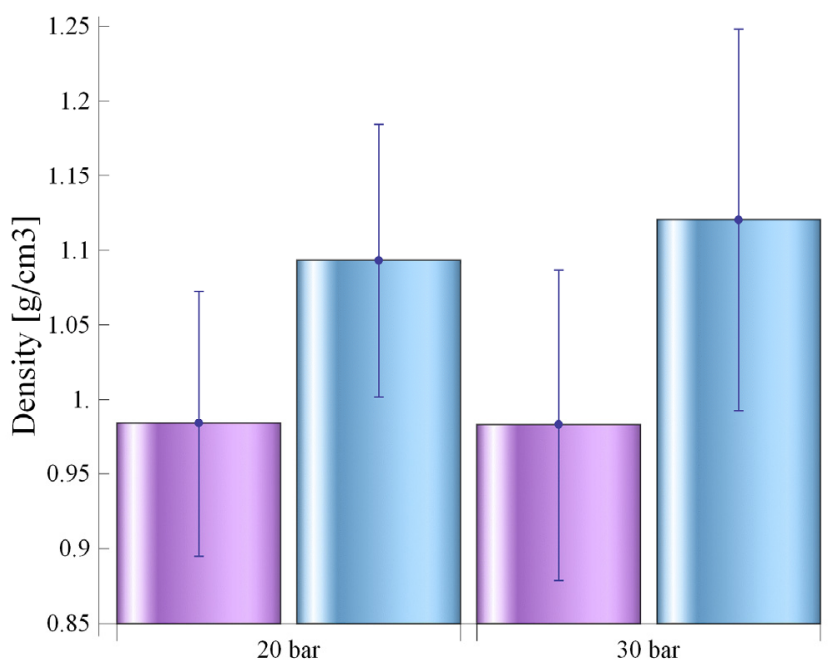

Figure 5: Foam density according to temperature and pressure variations on two levels. The error bars correspond to the standard deviation.

In order to find the relationship between manufacturing process parameters and foam morphology, image analysis has been used to study specimen longitudinal sections. Void distribution and averaged density as well as void circularity, elongation and rectangularity have been analysed and their mean values have been reported in Tab. 2.

Specimen \#12 has been evaluated as an outlier sample since its external wall is strongly discontinuous and moreover its section is affected by anomalous cavities, probably due to inefficient infiltration through the salt pattern (Fig. 6). This is confirmed by standardised residual (the ratio of residual to the estimate of its standard deviation) of this specimen because its value, 2.11, is greater than 2, thus it must be classified as an unusual observation [20]. In figure 6 it is evident 
how the voids in specimen \#12 are unevenly distributed and of greater size than desired, and in the specimen centre part they also are merging together to create a single great cavity.

\begin{tabular}{ccccc}
\hline Specimen & $\begin{array}{c}\text { averaged } \\
\text { void } \\
\text { density }\end{array}$ & circularity & elongation & rectangularity \\
$\# 1$ & 0.676 & 0.612 & 0.437 & 0.483 \\
$\# 2$ & 0.581 & 0.600 & 0.434 & 0.459 \\
$\# 3$ & 0.666 & 0.602 & 0.415 & 0.489 \\
$\# 4$ & 0.661 & 0.603 & 0.443 & 0.462 \\
$\# 5$ & 0.589 & 0.584 & 0.423 & 0.481 \\
$\# 6$ & 0.667 & 0.608 & 0.451 & 0.488 \\
$\# 7$ & 0.645 & 0.585 & 0.414 & 0.479 \\
$\# 8$ & 0.563 & 0.599 & 0.443 & 0.469 \\
$\# 9$ & 0.515 & 0.577 & 0.416 & 0.480 \\
$\# 10$ & 0.568 & 0.560 & 0.411 & 0.475 \\
$\# 11$ & 0.535 & 0.570 & 0.442 & 0.474 \\
$\# 12$ & 0.738 & - & - & - \\
mean & 0.617 & 0.591 & 0.430 & 0.476 \\
std. dev. & 0.068 & 0.017 & 0.014 & 0.010 \\
\hline
\end{tabular}

Table 2: Morphological indicators of the investigated sections (mean values).
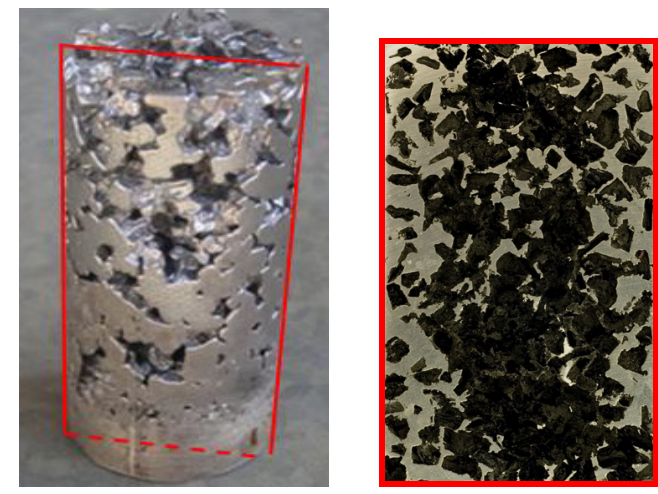

Figure 6: Section of specimen \#12.

Concerning the morphological indicators (circularity, elongation and rectangularity) standard deviations are less than $4 \%$. The correspondent ANOVA, made with a 95\% confidence interval on circularity, reveals a significant single effects of temperature and a less remarkable effect of the pressure, while no significant effects on the other indicators are recognised through the ANOVA calculations on elongation and rectangularity values.

Standard deviation of the averaged void density shows the highest value (about 11\%). Two factors can affect this variation: the local distribution of void density along the investigated section and the variation of the process parameters. Fig. 7 shows the averaged void density according to temperature and pressure variations. The results are consistent with the foam density graphs reported in Fig. 5. In fact when the temperature level increases the averaged void density decreases due to a higher foam density.

Pressure effects on void density seem to be slightly different from the results achieved in Fig. 5 probably due to a major role played by the local distribution of voids on the sections. To better asses this matter in Fig. 8 the normalised occurrence histograms of the local distribution of foam density ( $\mathrm{FD}=1$-void density $_{\text {) }}$ are shown. The density maps have been computed by considering the grey level image's array of pixel values, i.e. a matrix of real values scaled to the range 0 to 1 . 
Each point of the maps is obtained calculating the mean of a moving fragment of this matrix corresponding to a square 4 $\mathrm{mm}$ in length, in order to not consider single void variation.

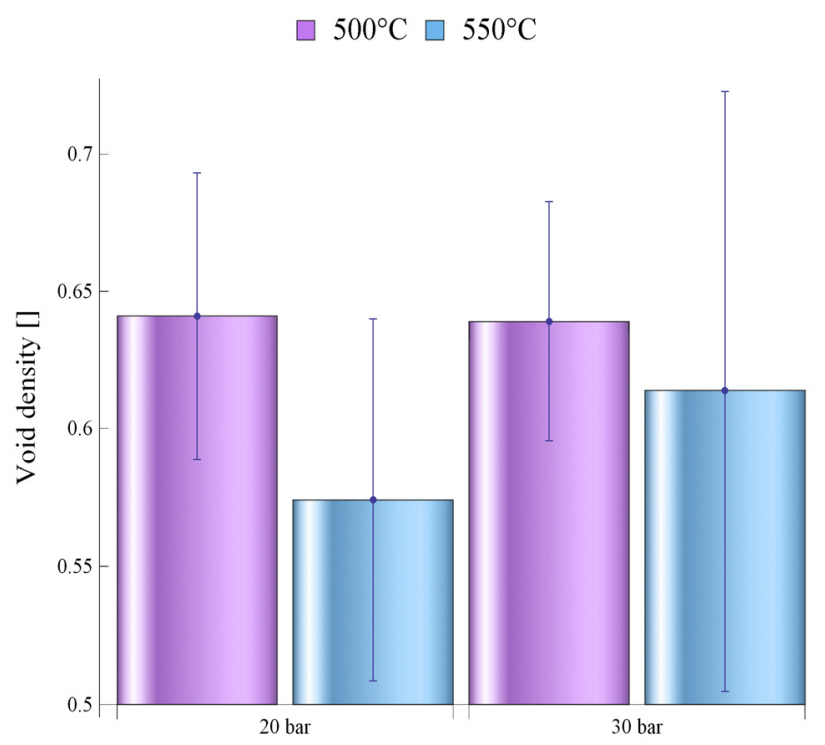

Figure 7: Averaged void density according to pressure and temperature variation on two levels. The error bars correspond to the standard deviation.

Fig.8 shows that the specimens, in which the salt particles are well replicated and the cavities are spread regularly, have symmetric frequency histograms (\#2, \#5, \#8, \#9, \#10, \#11). Conversely specimens \#1, \#3, \#4, \#6, \#7, \#12 present large localized cavities and their frequency histograms are asymmetric.

From the analysis of the macrographs it can be observed that specimens obtained with a $550^{\circ} \mathrm{C}$ preheated mould exhibit more regular cavities distribution; among them, the ones infiltrated at 30 bar are the most uniform. This confirms the assumption that higher pressure coupled with higher temperature optimises metal infiltration.

The interaction between temperature and pressure to improve the infiltration is also investigated by analysing void circularity. As shown in Fig.9a the void circularity at the lower mould temperature does not depend upon the applied pressure (as suggested by standard deviations); at the higher preheating temperature as the pressure increases the circularity decreases. Moreover at low and high pressure levels the temperature increase determines the reduction of the circularity. It can be noticed that for the set parameters $550^{\circ} \mathrm{C}$ and 30 bar the lowest circularity value (0.565) is achieved. This behaviour can be explained considering that in these conditions the liquid metal infiltrates more effectively the salt bed and therefore the salt particles are well replicated and cavities tend to become less rounded (generally speaking circularity of 0.886 is related to square shape, while 0.709 is the circularity of the rectangular shape with an aspect ratio of 1:4). As already said introducing table 2 the ANOVA analysis confirms that temperature has a significant effect on the circularity with a confidence interval of $95 \%$, while there is not evidence for pressure effect.

As far as elongation (Fig.9b) and rectangularity (Fig.9c) values are concerned they appear to be insensitive to changes of process parameters. Voids seem slightly more elongated only when metallic foam is produced by applying 30 bar pressure and by preheating the mould at $500{ }^{\circ} \mathrm{C}$. This is probably due to the fact that, in these operative conditions, there is a considerable chill from the mould face. The consequence is that the alloy is characterised by a poor fluidity and it is pushed by the piston through the salt bed producing more elongated cavities. As far as the rectangularity is concerned, it can be noticed that the specimens produced with the set parameters $550^{\circ} \mathrm{C}$ and 30 bar have a standard deviation markedly smaller than the other sets. The small sensitivity of these indicators to process parameters can be probably due to the shape of the salt pattern adopted during the manufacturing process.

A further aspect that needs to be analysed is the effect of process parameters on the alloy microstructure. The microstructure of the alloy is typical of a hypoeutectic Al-Si alloy, with primary $\alpha$-Al dendrites and eutectic Si particles distributed around the $\mathrm{Al}$ dendrites to form a cell pattern periodically repeated across the surface. 

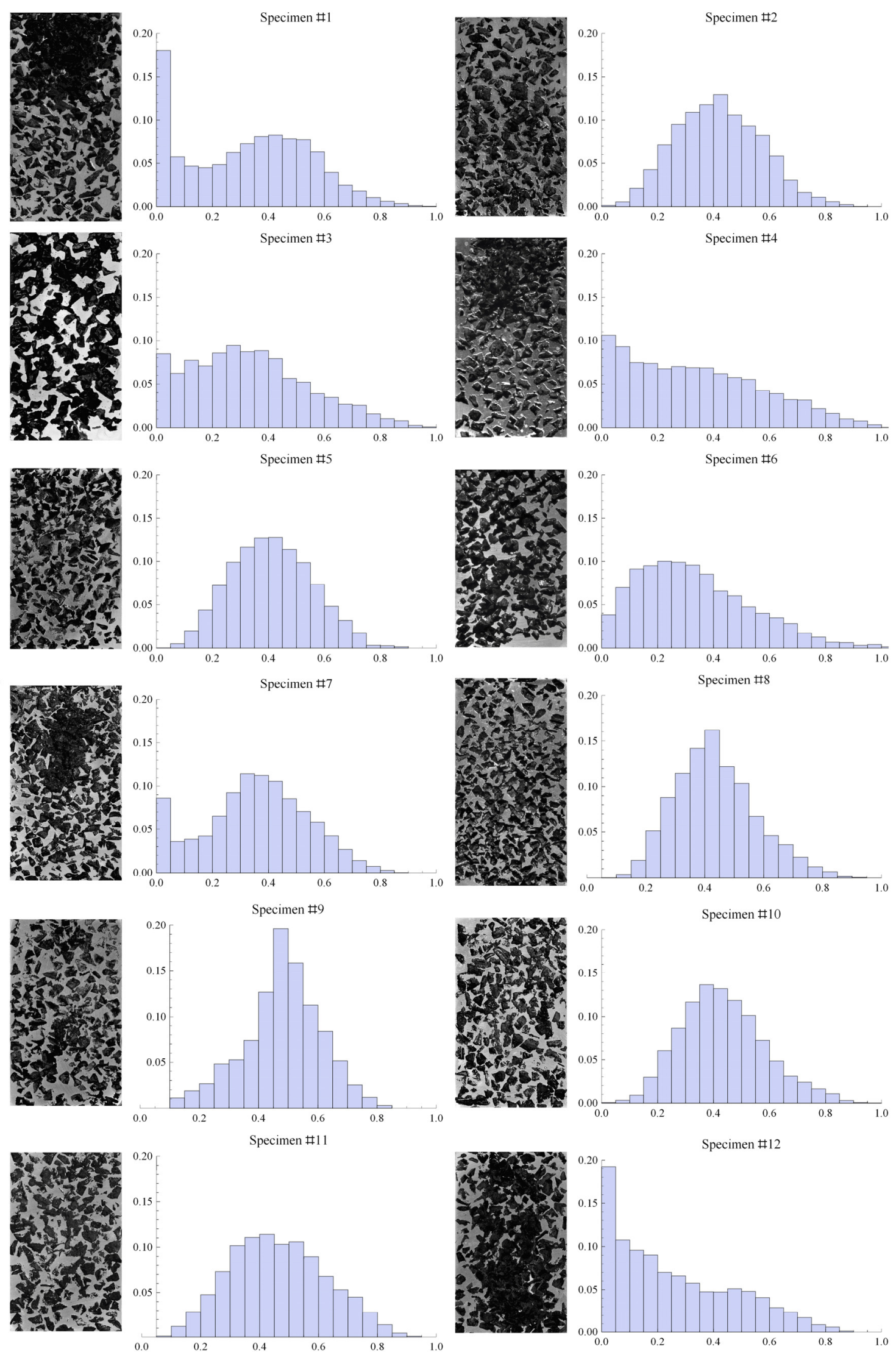

Figure 8: Specimens macrographs and normalised occurrence histograms of FD. 

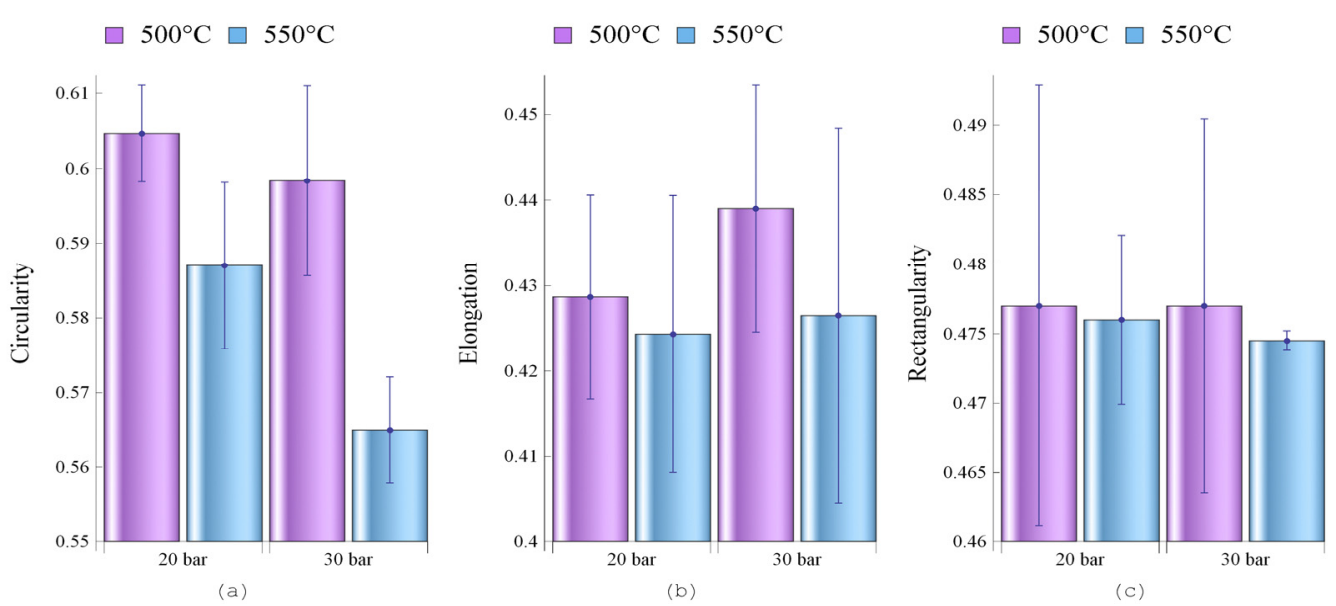

Figure 9: Circularity (a), Elongation (b) and Rectangularity (c) according to temperature and pressure variations on two levels. The error bars corresponds to the standard deviation.

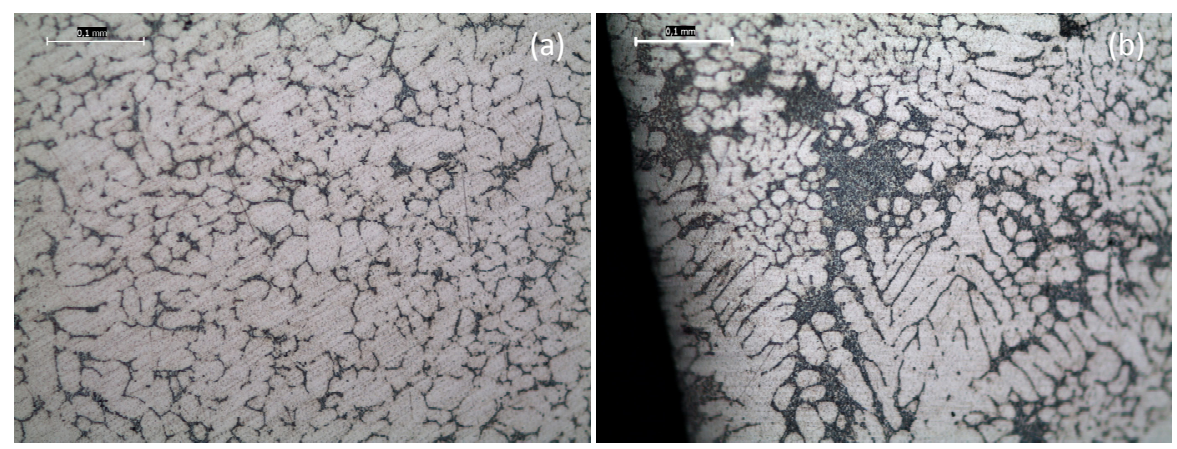

Figure 10: Optical micrographs showing the microstructure of specimen \#1 (a) and of an area close to a void in specimen \#2 (b).

Microstructural analysis highlighted that the microstructure of alloys cast in different operative conditions are very similar to each other. The specimens showing a finer structure (Fig. 10) are only the ones produced by preheating the mould at $550{ }^{\circ} \mathrm{C}$ and by applying a pressure of 30 bar. In those conditions the melt infiltrates efficiently the salt pattern and there is a considerable chill from the salt surface that locally increases cooling rates. By analyzing the foam microstructure it is also apparent that the most heterogeneous areas are the ones close to the voids where solidification conditions are far away from equilibrium. Those areas are richer of eutectic Si. As far as the eutectic Si particles are concerned the used manufacturing process, which is characterized by very high cooling rates, determines the formation of rounded and short lamellae that improve the mechanical properties of the alloy. A close examination of silicon and intermetallic phases, carried out on deep etched specimens, highlighted that both of them may have platelet and rod-like morphology (Fig. 11).
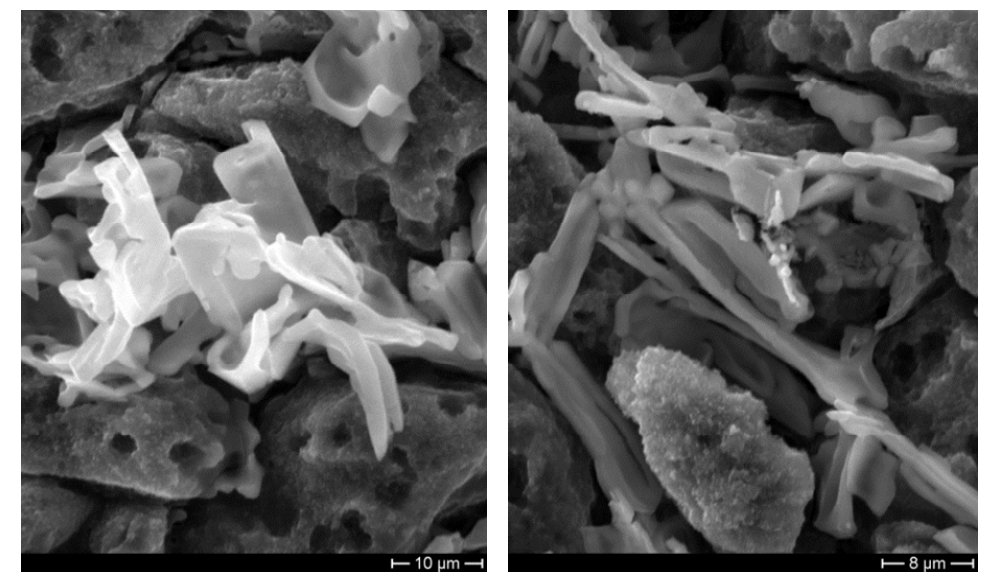

Figure 11: SEM micrographs showing the morphology of a silicon (a) and of an iron-rich intermetallic particle (b). 


\section{CONCLUSIONS}

$\mathrm{I}$ $\mathrm{n}$ this work replicated $\mathrm{AlSi} \mathrm{Mg} 0.3$ alloy foams are produced by infiltrating preforms of $\mathrm{NaCl}$ particles, varying the metal infiltration pressure and the mould temperature. An original procedure based on image analysis has been set up to determine size, morphology and distribution of cells. The results highlighted that when the mould is preheated at $500{ }^{\circ} \mathrm{C}$ there is an excessive chill and the liquid metal does not develop a smooth skin through intimate contact with the mould surface. When the mould is preheated at $550{ }^{\circ} \mathrm{C}$ the void density decreases by increasing the applied pressure. Moreover an increased infiltration pressure enhances metal infiltration through the salt pattern. In these conditions the void density distribution is more regular and there is a more effective replication of salt particles with a consequent reduced void circularity. This confirms the assumption that higher infiltration pressure coupled with higher mould preheating temperature optimises metal infiltration. The proposed procedure used to analyse the foam sections appears an effective way to analyse the effect of process parameters on foam morphology.

\section{REFERENCES}

[1] Ashby, M.F., Evans, A.G., Fleck, N.A., Gibson, L.J., Hutchinson, J.W., Wadley, H.N.G., Metal Foams: A Design Guide, Edited by Butterworth-Heinemann, USA (2000).

[2] Degischer, H.P., Kriszt B., Handbook of Cellular Metals: Production, Processing, Applications, Edited by H.P. Degischer, and B. Kriszt, Austria (2003).

[3] Polonsky, L., Lipson, S., Markus, H., Light weight cellular metal, Mod. Cast., 39 (1961) 57-71.

[4] Al-Jibbouri, S., Ulrich, J., The influence of impurities on crystallization kinetics of sodium chloride, Cryst. Res. Technol., 36 (2001) 1365-1375.

[5] Adair, J.H., Suvaci, E., Morphological control of particles, Curr. Opin. Colloid In., 5 (2000) 160-167.

[6] Gaillard, C., Despois, J.F., Mortensen, A., Processing of NaCI powders of controlled size and shape for the microstructural tailoring of aluminium foams, Mat. Sci. Eng. A-Struct., 374 (2004) 250-262.

[7] Jiang, B., Zhao, N.Q., Shi, C.S., Li, J.J., Processing of open cell aluminum foams with tailored porous morphology, Scripta Mater., 53 (2005) 781-783.

[8] Goodall, R., Marmottant, A., Salvo, L., Mortensen, A., Spherical pore replicated microcellular aluminium: Processing and influence on properties, Mat. Sci. Eng. A-Struct., 465 (2007) 124-135.

[9] Goodall, R., Despois, J.F., Marmottant, A., Salvo, L., Mortensen, A., The effect of preform processing on replicated aluminium foam structure and mechanical properties, Scripta Mater., 54 (2006) 2069-2073.

[10] Despois, J.F., Marmottant, A., Salvo, L., Mortensen, A., Influence of the infiltration pressure on the structure and properties of replicated aluminium foams, Mat. Sci. Eng. A-Struct., 462 (2007) 68-75.

[11] Kádár, C., Chmelík, F., Cieslar, M., Lendvai, J., Acoustic emission of salt-replicated foams during compression, Scripta Mater., 59 (2008) 987-990.

[12] Quadrini, F., Boschetto, A., Rovatti, L., Santo, L., Replication casting of open-cell AlSi7Mg0.3 foams, Mater. Lett., 65 (2011) 2558-2561.

[13] Boschetto, A., Costanza, G., Quadrini, F., Tata, M. E., Cooling rate inference in aluminum alloy squeeze casting, Mater. Lett., 61 (2007) 2969-2972.

[14] Boschetto, A., Campana, F., Giordano, V., Pilone, D., Morphological characterisation of cellular materials by image analysis, In: P. Di Giamberardino, D. Iacoviello, M. João (Eds), Computational Modelling of Objects Represented in Images III: Fundamentals, Methods and Applications, Italy (2012) 391-396.

[15] Banhart, J., Manufacture, characterisation and application of cellular metals and metal foams, Prog. Mater. Sci. 46 (2001) 559-632.

[16] Baumeister, J., Banhart, J., Weber, M., Aluminium foams for transport industry, Mater. Design, 18 (1997) 217-220.

[17] Vaidya, U.K., Pillay, S., Bartus, S., Ulven, C.A., Growc, D.T., Mathewc, B., Impact and post-impact vibration response of protective metal foam composite sandwich plates, Mat. Sci. Eng. A-Struct., 428 (2006) 59-66.

[18] Campana, F., Pilone, D., Effect of wall microstructure and morphometric parameters on the crush behaviour of Al alloy foams, Mat. Sci. Eng. A-Struct., 479 (2008) 58-64.

[19] Russ, J.C., The Image Processing Handbook, 5th ed., CRC Press Taylor \& Francis Group, USA (2006). 
[20] Montgomery, D.C., Runger, G.C., Applied Statistics and Probability for Engineers, 3rd ed., John Wiley \&Sons Inc., New Jersey (2003).

\section{VIDEO REFERENCES}

[V1] http://www.gruppofrattura.it/video/FIS26/Pilone/V1.mp4 